Purdue University

Purdue e-Pubs

2010

\title{
Microtomography-Based Simulation of Transport Through Open-Cell Metal Foams
}

Karthik K. Bodla

Purdue University - Main Campus

Jayathi Y. Murthy

Purdue University - Main Campus

S V. Garimella

Purdue University, sureshg@purdue.edu

Follow this and additional works at: http://docs.lib.purdue.edu/coolingpubs

Bodla, Karthik K.; Murthy, Jayathi Y.; and Garimella, S V., "Microtomography-Based Simulation of Transport Through Open-Cell Metal Foams" (2010). CTRC Research Publications. Paper 149.

http://dx.doi.org/10.1080/10407782.2010.511987

This document has been made available through Purdue e-Pubs, a service of the Purdue University Libraries. Please contact epubs@purdue.edu for additional information. 


\title{
Microtomography-Based Simulation of Transport through Open-Cell Metal Foams
}

\author{
Karthik K. Bodla, Jayathi Y. Murthy, Suresh V. Garimella*, \\ School of Mechanical Engineering, Purdue University, West Lafayette, IN 47907 USA
}

Running Title: Simulation of Transport through Open-cell Metal Foams

Keywords: microtomography, CT-scan, metal foam, effective conductivity, tortuosity, permeability, porous media

* Corresponding author, Email: sureshg@purdue.edu, Tel: 765-494-5646 


\section{Abstract}

Important heat transfer parameters of aluminum foams of varying pore sizes are investigated through CT-scanning at 20 micron resolution. Small sub-samples from the resulting images are processed to generate feature-preserving, finite-volume meshes of high quality. All three foam samples exhibit similar volumetric porosity (in the range $\sim 91-93 \%$ ), and thereby a similar thermal conductivity. Effective tortuosity for conduction along the coordinate directions is also calculated. Permeability simulations in the Darcy flow regime with air and water show that the foam permeability is isotropic and is of the order of $10^{-7} \mathrm{~m}^{2}$. The convective heat transfer results computed for this range of Reynolds numbers exhibit a dependence on the linear porosity, even though the corresponding volumetric porosity is the same for all the samples considered. 


\section{NOMENCLATURE}
A area, $\mathrm{m}^{2}$
C specific heat, $\mathrm{Jkg}^{-1} \mathrm{~K}^{-1}$
in inlet
$\mathrm{C}_{\mathrm{E}} \quad$ Ergun's coefficient
K permeability
f friction factor
1 linear
$\mathrm{h}$ heat transfer coefficient, $\mathrm{Wm}^{-2} \mathrm{~K}^{-1}$
mod modified
$\mathrm{J}$ diffusion flux vector, $\mathrm{Wm}^{-2}$
$\mathrm{r} \quad$ reference
$\mathrm{k}$ thermal conductivity, $\mathrm{Wm}^{-1} \mathrm{~K}^{-1}$
s solid
$\mathrm{K}$ permeability, $\mathrm{m}^{2}$
$\mathrm{T}$ total, pore and metal region
L length, $\mathrm{m}$
$\mathrm{v} \quad$ volumetric
n number of pores
water water, in the pore region
$\mathrm{P}$ pressure, $\mathrm{Nm}^{-2}$
Pr Prandtl number
q" heat flux, $\mathrm{Wm}^{-2}$
Re Reynolds number
s length of the curve, $m$
$\mathrm{u}, \mathrm{v}, \mathrm{w}$ velocities along $\mathrm{x}, \mathrm{y}, \mathrm{z}$ directions,

$$
\mathrm{ms}^{-1}
$$
$\mathrm{x}, \mathrm{y}, \mathrm{z} \quad$ Cartesian coordinate directions
$\mathrm{V}$ volume, $\mathrm{m}^{3}$

\section{Greek symbols}
$\varepsilon \quad$ porosity
$\rho$ density, $\mathrm{kgm}^{-3}$
$\mu \quad$ dynamic viscosity, $\mathrm{Nsm}^{-2}$
$\tau \quad$ tortuosity

\section{Superscripts}

$$
\text { - average }
$$

Subscripts
air air, in the pore region
eff effective
f fluid 


\section{INTRODUCTION}

X-ray tomography or computed tomography (CT) scanning is a popular visualization technique used for medical applications such as tumor and crack detection in bones [1]. It is based on the differential absorptivities of different materials to X-rays. The object to be scanned is irradiated with X-rays and the resulting reflected beam is reconstructed using a set of algorithms such as fan beam reconstruction and cone beam reconstruction [2]. The differential absorptivity (related to the material density) is reflected in the reconstructed images through regions of varying grayscale values. Distinction based on a simple threshold can then be made to identify the different materials in the original object. This technique has traditionally been limited to non-destructive analysis of live samples, but is steadily gaining importance in the field of materials testing, with the advent of superior reconstruction algorithms and powerful cameras with sub-micron scale resolution ( $\mu$-CT or X-ray microtomography (XMT)). Nanotomographs, with a resolution of the order of a few nanometers, are also used [3].

$\mathrm{XMT}$ is similar to CT in terms of the operating principle, but provides an image resolution on the scale of a few microns. Today, XMT is used for a wide range of applications ranging from materials research $[4,5]$ to precision reverse-engineering of complex parts [6]. With its micron-scale resolution, XMT can be used for visualizing the intricate details of materials such as porous media, which would be impossible with traditional destructive methods. Finite-element analysis of bones was performed in [7] based on XMT data. Tabor et al. [8] presented the idea of a voxel-based reconstruction method for generating finite-volume meshes from the $\mu$-CT data. The ability of the method to correctly represent the complex structure of porous media such as auxetic foams was demonstrated.

In the present work, we use the approach in the context of accurate flow and heat transfer analysis of porous media. Metal foams are novel materials with many desirable features such as low density and superior structural and thermal properties [9] owing to a large surface area to volume ratio. They have been shown $[10,11$,$] to provide an effective solution to many thermal problems.$

Extreme randomness restricts the study of foams to largely experimental methods. In terms of modeling, much of the work has been limited to employing a macroscopic or semi-empirical type approach $[12,13]$, where volume-averaged equations are used to describe flow characteristics. A microscopic or pore-based approach, on the other hand, is potentially more accurate because local geometric information can be preserved. Various idealized periodic cell representations of metal foams, such as cubic unit cells consisting of square cylinders [14] or BCC cell models [15], have been used to capture microscale effects. Though these models have been very useful in understanding porous medium behavior, the models are generally valid only for a subset of the porosity range because of the approximate representation of geometry. In the present work, the aim is to use geometrically faithful, 
pore-scale feature-preserving meshes to correctly represent the foam sample under study, without any approximations regarding geometry. Although the model is sample-specific, it serves to establish that an accurate representation of geometry is necessary to produce accurate results for flow and heat transfer properties in complex porous media. The following sections describe the mesh generation procedure and the results obtained.

\section{FOAM GEOMETRY AND MESH GENERATION}

In this section, the image processing and mesh generation methods are briefly described along with the tools employed.

\subsection{Sample preparation}

With no approximations introduced in the governing equations, pore-scale microscopic modeling of porous media is computationally very expensive, which limits the sample sizes that can be analyzed using this technique. Moreover, most commercial scanners have a trade-off between the size of the samples being scanned and the scan resolution. In this work, samples of size $10 \mathrm{~mm}$ x $12.7 \mathrm{~mm}$ x $38.1 \mathrm{~mm}$ are cut from the original foam slabs using EDM (electric discharge machining), to ensure that the cuts are clean and that the cut samples are not distorted. The samples are then imaged using axial $\mu-\mathrm{CT}$ at 20 micron resolution employing a commercial X-ray scanner- $\mu \mathrm{CT} 40$ from SCANCO MEDICAL, with the axis along the longer dimension. The resolution is selected such that the ligaments are properly reconstructed in the image reconstruction step and that all other microscale features, such as the pore orientation and shape, are well-represented. The scanner software generates a $2 \mathrm{D}$ stack of images corresponding to the 3D object scanned, which can be later analyzed after surface/volume reconstruction.

\subsection{Image processing and mesh generation}

The resulting images from the scan data must be processed before they can be used for mesh generation. The details are described only briefly here and a more detailed description may be found in [16].

The image processing is done using the module ScanIP of the commercial software Simpleware [16]. The software has many features such as noise removal, region identification based on grayscale value, and 3D surface/volume reconstruction, which can later be exported in many standard formats for CAD or mesh generation. The scanned images are generally not sharp and the noise in each image can be rectified using the noise reduction filters provided by the module. The 'metal artifact reduction' filter with parameters as described in [16] is employed here. Once the images are clean, separate regions corresponding to the metal and the pore domains must be identified. The distinction is based on the 
grayscale value. The metal is much denser than the surrounding pore region, which is filled with air. It absorbs X-rays, while the pore region lets the X-rays pass through. This difference is reflected in the reconstructed image stack, where the brighter regions correspond to the metal and the darker regions to the surrounding pore. But there is no smooth transition, and hence the detection is based on a threshold value selected to ensure that the porosity of the reconstructed sample is the same as the original sample. The identified regions are then 'stitched' with unit pixel separation among every two adjacent images in the $2 \mathrm{D}$ image stack to generate $3 \mathrm{D}$ volumes corresponding to the metal and pore domains of the original foam sample. After this, the model is checked to ensure that there are no unconnected regions or islands; those that exist are removed using the 'floodfill' segmentation tool. At this point, the processed data are analyzed for the number of pixels contained, which are directly related to the number of volumes in the finite-volume mesh. A large number of pixels not only increases the number of cells in the mesh, but also the demand on memory of the mesh generating module, ScanFE, which is limited. Careful downsampling is hence performed such that the foam geometry is still well-represented and only smaller subvolumes are considered for meshing. The various image processing steps are illustrated in Figure 1.

The processed data are then input to the ScanFE module for generating finite-volume meshes. The processed data at this stage consist of voxels or 3D pixels, which if directly used for meshing, would generate a stair-stepped mesh. Therefore the data are first smoothed and finite-volume meshes with tetrahedral elements of very high quality are generated. The mesh thus produced is exported to the FLUENT solver [17], which is used for flow and heat transfer analysis. The metal and pore regions, and an inset of the mesh, are shown in Figure 2. ScanFE ensures that the smoothing process preserves mesh conformality and good element quality.

\section{NUMERICAL MODELING}

This section describes the governing equations and boundary conditions and other modeling details such as discretization schemes employed and convergence criteria.

\subsection{Governing equations and boundary conditions}

The continuity, momentum and energy equations for the steady constant-property flow of an incompressible Newtonian fluid are given by [18]:

$$
\begin{gathered}
\frac{\partial}{\partial x_{i}}\left(\rho u_{i}\right)=0 \\
\frac{\partial}{\partial x_{j}}\left(\rho u_{j} u_{i}\right)=-\frac{\partial P}{\partial x_{i}}+\mu\left(\frac{\partial^{2} u_{i}}{\partial x_{j} x_{j}}\right)
\end{gathered}
$$




$$
\frac{\partial}{\partial x_{i}}\left(\rho C u_{i} T\right)=\frac{\partial}{\partial x_{i}}\left(k \frac{\partial T}{\partial x_{i}}\right)
$$

A cuboidal porous block is chosen as the computational module, with symmetry boundary conditions on the lateral boundaries, i.e., on block faces parallel to the mean flow direction. Two types of simulations are performed. The first involves the computation of effective thermal conductivity of the porous sample in the presence of an interstitial fluid. Here, a conjugate conduction problem is solved in the domain with an imposed temperature gradient across the domain. The second type of simulation involves the computation of pressure drop and heat transfer coefficient. For these simulations, a no-slip boundary condition is employed on the interface that separates the solid and fluid regions. A pressure outlet boundary condition is employed at the outlet, while a velocity inlet boundary condition is employed at the inlet (Figure 3). Though a fully developed flow assumption is not made, we found that the crosssectionally averaged pressure achieved a linear profile within the sample for all the Reynolds numbers considered here. For the computation of Nusselt number, conduction in the solid is not considered, and a constant heat flux boundary condition is imposed at the solid-fluid interface. As with the pressure drop computation, we did not explicitly impose a fully developed thermal condition; nevertheless, the Nusselt number was found to become constant in the flow direction for the sample sizes, flow conditions and fluids considered here.

\subsection{Solution procedure}

For the domain sizes concerned, computational meshes of about 15 million cells are generated using the procedure described in the previous section. The governing equations are solved using the commercial solver FLUENT [17], using a second-order upwind scheme for the flow and heat transfer calculations. Pressure-velocity coupling is addressed using the SIMPLE algorithm, along with an algebraic multigrid algorithm for solving the linearized system of governing equations. Details of the numerical method may be found in [17]. The governing equations are suitably under-relaxed to ensure proper convergence. The default convergence criterion in FLUENT, based on the scaled residuals, is employed for termination.

It may be noted that the mesh sizes employed in the present work are dependent on the image resolution as described in section 2 . As the image resolution is changed to change the mesh size, the porosity of the samples also changes. Therefore, a fixed resolution is used, and a mesh-independence study is performed in an indirect manner. The average number of pores per sample for the domain sizes considered in this study is $\approx 150$, corresponding to an average mesh size per pore of 0.1 million cells. Based on the grid-independence study performed by Krishnan et al. [15], who observed a 2.6\% deviation 
with a mesh size per pore of 106,520 cells relative to the finest mesh of 383,230 cells, results in the present work are considered to be reasonably independent of the mesh size.

\section{RESULTS AND DISCUSSION}

The porosity, thermal conductivity, tortuosity, friction factor and heat transfer coefficient results for the three foam samples are presented in this section.

\subsection{Porosity and thermal conductivity}

The porosity of a porous medium may be defined in a number of ways. Commercially, the various foam samples made out of the same metal are distinguished by defining a linear porosity $\varepsilon_{l}$ as:

$$
\varepsilon_{l}=\frac{n}{L}
$$

Here, $n$ is the number of pores and $L$ is the length of the sample. It is expressed in pores per inch (ppi); hence a 10 ppi foam sample has, on average, 10 pores per inch of the sample. Another more practical definition, volumetric porosity $\varepsilon_{v}$, is based on the volume ratio:

$$
\varepsilon_{v}=\frac{V_{f}}{V_{T}}
$$

In Eq. (5), $V_{f}$ is the pore volume and $V_{T}$ is the total volume. For the samples under consideration, it is observed that though the linear porosity $\varepsilon_{l}$ was different, all the foam samples exhibited a similar volumetric porosity, $\varepsilon_{v}$ in the range $93 \pm 1.5 \%$. That is, samples with different linear porosity have different pore sizes, but the foam-ligament thickness changes from sample to sample to keep the volumetric porosity more or less the same. This can be observed in Figure 4.

For computing thermal conductivity in direction $x_{i}(\mathrm{x}, \mathrm{y}$ or $\mathrm{z})$, a temperature gradient $\left(\frac{\partial T}{\partial x_{i}}\right)$ is imposed across the two ends of the cuboidal sample, while the lateral faces are held adiabatic. No pressure gradient is imposed; the pores are filled with fluid, but the fluid is static. The effective thermal conductivity, $k_{\text {eff }}$, is calculated as:

$$
k_{e f f}=\frac{-\int \mathbf{J} \cdot d \mathbf{A}}{\left(\frac{\partial T}{\partial x_{i}}\right) A}
$$


$\boldsymbol{J}$ is the heat flux vector and $d \boldsymbol{A}$ is the outward pointing area vector. It is to be noted that the effective thermal conductivity is calculated by considering conduction through both the solid and fluid regions, i.e., the integral in Eq. (6) is performed on both the solid and fluid regions of the face, and $A$ is the total conduction area. The temperature contours for the 10 ppi foam sample are shown in Figure 5. The simulations for thermal conductivity are performed by considering only half of the scanned samples, i.e., regions of size $10 \mathrm{~mm}$ x $12.7 \mathrm{~mm}$ x $19 \mathrm{~mm}$, due to memory limitations in ScanIP and ScanFE for mesh generation.

It was observed that the effective thermal conductivities were different in the three coordinate directions, and certain samples showed up to a $15 \%$ deviation. The primary reason for this is that the computational domain contains only a few pores for the cases computed here. The number of pores in the longest direction is between 15 and 25 and along the smallest direction is between 3 and 6 , depending on the foam sample (10 ppi, 20 ppi or $40 \mathrm{ppi}$ ). However, computational limitations on mesh size preclude the employment of larger sample sizes. Hence, we report an effective thermal conductivity averaged over the three co-ordinate directions. The average effective thermal conductivity values for the three foam samples, with air or water as the interstitial fluid, along with their respective porosities, are tabulated in Table 1. The computations show that the thermal conductivity is approximately the same for the three samples, indicating that the primary dependence is on the volumetric porosity rather than on the pore size.

Figure 6 shows a comparison of the present simulations with experimental values [13], and semiempirical models from the literature [12-13,19]. Also plotted are the results from Krishnan et al. [15], who developed an open-cell model for aluminum foams which predicted good values for foams with volumetric porosities greater than 0.94 .

Further, results employing the Lemlich theory $[20,15]$ are shown, where the effective thermal conductivity, exploiting the analogy between heat conduction and electrical conduction, is defined as:

$$
k_{\text {eff }}=k_{s} \frac{\left(1-\varepsilon_{v}\right)}{3}
$$

Figure 6 shows that the present model is able to predict the effective thermal conductivity well, even at lower porosities. It may be noted that the models of Boomsma and Poulikakos [12], Calmidi and Mahajan [13], and Bhattacharya et al. [19], employ a free parameter to match the experiments of Calmidi and Mahajan [13]. In the current model, no such parameter has been employed and the effective thermal conductivity is directly computed using a correct description of the foam geometry. Another observation regarding the Lemlich theory may be made from Figure 6 . The theory predicts the effective thermal conductivity fairly well when the interstitial fluid is air, while with water, the error with respect to the experimental values [15] is greater. The results indicate that larger sample sizes must be used in the 
computation to mitigate the effect of domain-size artifacts, and multiple realizations of the sample geometry are necessary to obtain statistically invariant predictions in these random media. These caveats also apply to experimental measurements. Nevertheless, the match between model predictions and data is encouraging.

\subsection{Tortuosity}

In averaging the thermal conductivity in the three coordinate directions, we have assumed that the porous structure is isotropic. One measure of whether this assumption is valid is the tortuosity. The tortuosity of a curve may be defined as:

$$
\tau=\frac{s}{L_{r e f}}
$$

Here $s$ is the length of the curve and $L_{r e f}$ is a reference length, usually the distance between the end points. Tortuosity is the measure of a curve's deviation from being straight. If the conduction paths in one direction, say $\mathrm{x}$, are more tortuous than the paths in another direction, say $\mathrm{y}$, the material would exhibit a higher thermal conductivity in direction y, all other parameters being the same. This parameter is widely employed for analyzing blood flows through arteries [21] and also in other porous media studies such as in hydrogeology [22]. In this work, we use $\tau$ to characterize the anisotropy of the foam sample. The average tortuosity for the conduction paths (foam ligaments) along the three co-ordinate directions is reported (Table 2), with the reference length being the length of the sample in that direction, i.e. $12.7 \mathrm{~mm}$ in $\mathrm{x}, 10 \mathrm{~mm}$ in $\mathrm{y}$ and $19 \mathrm{~mm}$ in $\mathrm{z}$ direction.

The tortuosity is calculated by constructing a nodal network of the foam samples from the corresponding 'image skeleton' using the commercial software Amira [23], and measuring the length of the corresponding conduction paths. An image skeleton or medial axis of an image is a skeletal remnant that largely preserves the extent and connectivity of the original region while discarding most of the original foreground pixels. Various definitions for the skeletons have been proposed in literature. One of the first [24] was based on a "grass fire" model, i.e., a moving wave-front generated by an inward motion of an outline curve with constant speed along a normal vector at every point on the curve. The skeleton is the set of points at which the wavefront crosses itself. It is widely employed for data reduction purposes in the fields of computer vision, medicine and pattern recognition. A 10 ppi foam sample along with a nodal network representation of its skeleton is shown in Figure 7.

The tortuosities shown in Table 2 are relatively invariant with respect to the linear porosity in the long direction of the sample (z). The tortuosity is more or less invariant with respect to direction, given 
the limitations of sample size and the fact that only a single realization of the sample was used in the characterization. Thus, we do not see indications that the sample is anisotropic.

Image skeletons of the type shown in Figure 7 may be used for other purposes as well. For example, approximate but very fast computations of effective solid thermal conductivity may be performed using the skeletal representation for applications in which the interstitial fluid has negligible thermal conductivity, such as air.

\subsection{Permeability}

Permeability, an important flow parameter which governs the pressure drop in a porous medium, is calculated here for the three foam samples. The results are reported for the Darcy flow regime $\left(\operatorname{Re}_{\mathrm{K}} \sim 10\right.$ or lower). For this computation, as described previously, a velocity inlet boundary condition is employed at the inlet while a pressure outlet boundary condition is used at the flow outlet. All other boundaries transverse to the main flow direction are made symmetric and a no-slip boundary condition is employed for the metal foam wall. The resulting pressure drop is then used to estimate the permeability employing Darcy's law [25]:

$$
\begin{gathered}
-\frac{\partial P}{\partial x_{i}}=\frac{\mu}{K} U_{\text {mod }}+\frac{\rho C_{E}}{\sqrt{K}} U_{\text {mod }}^{2} \\
U_{\text {mod }}=\frac{A_{f}}{A_{T}} U_{i n}
\end{gathered}
$$

$A_{f}$ is the average pore area, $A_{T}$ is the total cross-sectional area in a flow direction (x, y or z) and $C_{E}$ is the Ergun's coefficient. $U_{\text {mod }}$ is the modified inlet velocity, based on the total cross-sectional area of the foam sample. The pressure gradient is calculated considering only the fully developed region (identified as a region with a linear pressure gradient). Table 3 shows the permeability and Ergun's coefficient values for flow of air and water. It may be noted here that a sample size $\sim 10 \mathrm{~mm}$ in the flow direction was sufficient to achieve fully developed flow for the range of Reynolds numbers studied in this work. Hence, we use only a quarter of the original scanned sample, i.e., a sample of size $10 \mathrm{~mm}$ x $12.7 \mathrm{~mm}$ x $9.53 \mathrm{~mm}$ for the estimation of permeability, friction factor and heat transfer coefficient.

Permeability and Ergun's coefficient are calculated as least-squares fits to the pressure drop variation with inlet velocity and backing out the corresponding coefficients. The permeability, in contrast to thermal conductivity, was found to be largely isotropic. Moreover, different sub-sections from the original scanned sample exhibited similar permeability. Therefore, we report the permeability for the flow along the longest direction of the domain considered. It may be inferred from Table 3 that permeability is a direct function of pore size. The sample with the smallest pore size exhibits the smallest permeability and 
vice versa. Existing permeability correlations employ the porosity as the single geometric factor in the correlation [26]. The volumetric porosities corresponding to the three linear porosities in Table 3 are nearly the same, suggesting that other geometric features such as pore size may determine permeability. Experimental results for permeability of higher porosity foams than those considered here may be found in $[15,19]$.

\subsection{Friction factor and heat transfer coefficient}

The friction factor at a given Reynolds number may be estimated as follows [15]:

$$
f=\frac{\left(-\frac{\partial P}{\partial x_{i}}\right) \sqrt{K}}{\rho U_{\text {mod }}^{2}}
$$

$\left(-\frac{\partial P}{\partial x_{i}}\right)$ is the imposed pressure gradient in direction $x_{i}$. It may be noted that our definition of friction factor, Eq. (11), along with the definition of permeability from Eq. (9), leads to an alternate expression of the form:

$$
\begin{gathered}
f=\frac{1}{\operatorname{Re}_{K}}+C_{E} \\
\operatorname{Re}_{K}=\frac{\rho U_{\text {mod }} \sqrt{K}}{\mu}
\end{gathered}
$$

The friction factor data for the three foam samples along with the correlations of Paek et al. [27] and Vafai and Tien [28] are shown in Figure 8. It may be inferred from Figure 8 that friction factor scales as $1 / \mathrm{Re}_{\mathrm{K}}$ at lower Reynolds numbers, while at higher Reynolds numbers, the non-linear term $\mathrm{C}_{\mathrm{E}}$ begins to dominate because of the inertial effects. This deviation from linear to non-linear behavior is observed for $\operatorname{Re}_{\mathrm{k}} \sim>1$. In the current analysis, we have restricted ourselves to lower Reynolds numbers $\left(\operatorname{Re}_{\mathrm{K}}<4\right)$; higher Reynolds numbers will be considered in the future. This dependence has also been observed by [27] and [28] for low velocities, as shown in Figure 8. At higher Reynolds numbers, inertial effects become dominant and pressure drop is no longer a linear function of velocity. It may be noted that the correlation in [28] was developed for a foam metal with a permeability of $1.11 \times 10^{-7} \mathrm{~m}^{2}$, and only our 40 ppi foam sample, with permeability close to this value (Table 3), seems to agree with their correlation well, while the other two foam samples with higher permeabilities follow the Paek et al. correlation [27] with $<5 \%$ deviation. 
Heat transfer coefficients are computed by imposing a constant heat flux boundary condition and solving the energy equation in the fluid region using the converged flow solution. We define the average heat transfer coefficient based on the flow inlet temperature as:

$$
h=\frac{q^{\prime \prime}}{\bar{T}_{s}-T_{i n}}
$$

$\bar{T}_{s}$ is the average temperature of the solid foam wall at the imposed heat flux q". $\mathrm{T}_{\text {in }}$ is the fluid temperature at the inlet. The heat transfer coefficients for the 10 ppi, 20 ppi and 40 ppi foam samples are shown in Figure 9 (a) and (b), respectively, for the flow of air and water through the pore region. Typical temperature contours along with the flow field are shown for the 10 ppi foam sample in Figure 10. The corresponding Nusselt number data are shown in Figure 11, with Nusselt number defined as:

$$
N u=\frac{h \sqrt{K}}{k_{f}}
$$

All the foam samples perform similarly for $\operatorname{Re}_{\mathrm{K}}$ less than $\sim 2$ for the flow of air and $\operatorname{Re}_{\mathrm{K}}$ less than $\sim 1$ for the flow of water. This is as expected since at this lower range of $\operatorname{Re}_{\mathrm{K}}$ values, heat transfer is predominantly by conduction and it is similar for the three foam samples as they have a similar porosity. At higher Reynolds numbers, it is seen that the convection effects become dominant and deviations are observed between the three samples. The 40 ppi foam showed upto a $20 \%$ higher heat transfer coefficient for $\mathrm{Re}_{\mathrm{K}}>3$ with water as the working fluid. Thus, even though the volumetric porosity of the three samples is similar, differences of as much as $10 \%$ in the heat transfer coefficient are seen with pore size for air, and $20 \%$ for water at $\operatorname{Re}_{\mathrm{K}} \approx 2.2$. The smaller pore sizes yield the higher heat transfer coefficient, and the difference with respect to larger pore sizes is seen to increase with $\mathrm{Re}_{\mathrm{K}}$. This indicates that volumetric porosity is insufficient to characterize heat transfer in foams, particularly as inertial effects become more important, and pore size must be considered as well. The trade-off between large pressure drop and large heat transfer coefficients for the foam samples is also evident in Figure 9 and Figure 11.

\section{CONCLUSIONS}

Accurate microscale modeling of flow and heat transfer through porous media is performed employing feature-preserving, geometrically faithful meshes generated using $\mu$-CT and processed using image-based meshing. Three foam samples of varying linear porosity are considered for the detailed estimation of flow and heat transfer - volumetric porosity, effective thermal conductivity, permeability, friction factor and heat transfer coefficient. It is demonstrated that all the three samples exhibit a similar volumetric porosity in the range $93 \pm 1.5 \%$. The effective thermal conductivity is a strong function of the 
volumetric porosity, but is a much weaker function of the linear porosity. A directional dependence in the computed effective thermal conductivity is observed because of the small domain sizes employed. However, tortuosity computations indicate that the foam is essentially isotropic.

Permeability analysis for the Darcy flow regime establishes the inverse dependence of permeability on pore size. Friction factor results are validated with available correlations in literature. For the low Reynolds number range, they are observed to scale as $1 / \mathrm{Re}_{\mathrm{K}}$, while at higher Reynolds numbers, inertial effects are found to contribute. Computations of the heat transfer coefficient indicate that it is dependent on the linear porosity, i.e., the pore size of the sample, and variations of 10-20\% may be found (depending on the interstitial fluid), even for samples of the same volumetric porosity. An important conclusion of the work is that by decreasing the pore size, we may not expect much change in effective thermal conductivity for samples of the same volumetric porosity, while the heat transfer coefficient increases at the cost of a higher pressure drop.

\section{ACKNOWLEDGEMENT}

The authors acknowledge the assistance of Dr. Eric Nauman of Purdue University for kindly scanning the foam samples. Support for this work from industry members of Cooling Technologies Research Center, an NSF Industry/University Cooperative Research Center, is also gratefully acknowledged.

\section{REFERENCES}

[1] E. A. C. Johnson and P.G. Young, On the Use of A Patient-Specific Rapid-Prototyped Model to Simulate the Response of the Human Head to Impact and Comparison with Analytical and Finite Element Models, J. Biomech., vol. 38, pp. 39-45. 2005.

[2] L. A. Feldkamp, L. C. Davis, and J. W. Kress, Practical Cone-Beam Algorithm, J. Opt. Soc. Am., vol. A 1,pp. 612-619, 1984.

[3] C. A. Larabell, M. A. Le Gros, Biological Nano-Tomography, Proc. 8th Int. Conf. on X-ray Microscopy, IPAP Conference Series 7, pp. 239, Egret Himeji, Japan, 2005. 
[4] I. G. Watson, P. D. Lee, R. J. Dashwood, and P. Young, Simulation of the Mechanical Properties of an Aluminum Matrix Composite using X ray Microtomography, Metall. Mater. Trans., vol. A, 37A, pp. $551-558,2006$.

[5] D. Fularia, P. D. Lee, and D. Bernard, Determining the Fluid Flow and Deformation Behavior of Both Simulated and Measured Columnar-Dendritic Structures in Al-Cu Alloys, 5th Decennial Int. Conf. on Solidification Processing, 22-25 July, Sheffield. 2007.

[6] A. Flisch, J. Wirth, R. Zanini, M. Breitenstein, A. Rudin, F. Wendt, F. Mnich, and R. Golz, , Industrial Computed Tomography in Reverse Engineering Applications, Computerized Tomography for Industrial Applications and Image Processing in Radiology, 15-17, March Berlin, 1999.

[7] A. A. Aziz, C. Saury, and V. B. Xuan, On the Material Characterization of a Composite using Micro CT Image based Finite Element Modeling, Proc. of SPIE, vol. 16, pp. 32-39, 2006.

[8] G. Tabor, P. G. Young, T. Beresford, and Benatayallah, Mesh Construction from Medical Imaging for Multiphysics Simulation: Heat Transfer and Fluid Flow in Complex Geometries, J. Eng. App. Comp. Fluid Mech., vol. 1, pp. 126-135, 2007.

[9] Ashby M. F., Evans A., Fleck N. A., Gibson L.J, Hutchinson J. W., and Wadley H. J. G., Metal Foams: A Design Guide, Butterworth-Heinemann, Boston, 2000.

[10] K. Boomsma, D. Poulikakos, and F. Zwick, Metal Foams as Compact High Performance Heat Exchangers, J. Mech. Mater., vol. 35, pp. 1161-1176, 2003.

[11] R. Annapragada., S. V. Garimella, and J. Y. Murthy, Permeability and Thermal Transport in Compressed Open-celled Foams, Numer. Heat Transfer, vol. 54, pp. 1-22, 2008.

[12] K. Boomsma, and D. Poulikakos, On the Effective Thermal Conductivity of a Three-Dimensionally Structured Fluid-Saturated Metal Foam, Int. J. Heat Mass Transfer, vol. 44, pp. 827-836, 2001.

[13] V. V. Calmidi, and R. L. Mahajan, The Effective Thermal Conductivity of High Porosity Fibrous Metal Foams, ASME J. Heat Transfer, vol. 121, pp. 466-471, 1999. 
[14] P. DuPlessis, A. Montillet., J. Comiti, and J. Legrand, Pressure Drop Prediction for Flow Through High Porosity Metallic Foams, Chem. Eng. Sci., vol. 49, pp. 3545-3553, 1994.

[15] S. Krishnan, J. Y. Murthy, and S. V. Garimella, Direct Simulation of Transport in Open-Cell Metal Foam, ASME J. Heat Transfer, vol. 128, pp. 793-799, 2006.

[16] Simpleware Ltd., ScanIP, ScanFE and ScanCAD tutorial guide for SIMPLEWARE 3.1, 2009.

[17] Fluent Inc., User's Guide for FLUENT 6.0, 2002.

[18] F. M. White, Viscous Fluid Flow, McGraw Hill International, 1991.

[19] A. Bhattacharya, A. A. Calmidi, and R. L. Mahajan, Thermophysical Properties of High Porosity Metal Foams, Int. J. Heat Mass Transfer, vol. 45, pp. 1017-1031, 2002.

[20] R. Lemlich, A Theory for the Limiting Conductivity of Polyhedral Foam at Low Density, J. Colloid Interface Sci., vol. 64, pp. 107-110, 1978.

[21] E. Grisan, M. Foracchia, and A. Ruggeri, A Novel Method for Automatic Evaluation of Retinal Vessel Tortuosity, Proc. 25th Annual International Conference of the IEEE EMBS, Cancun, Mexico, 2003.

[22] Y. Watanabe, and Y. Nakashima, Two-Dimensional Random Walk Program for the Calculation of the Tortuosity of Porous Media, J. Groundwater Hydrol., vol. 43, pp. 13-22, 2001.

[23] AMIRA online user's guide for AMIRA 5, 2009.

[24] H. Blum, A Transformation for Extracting New Descriptors of Shape, in Wathen-Dunn, W., Models for the Perception of Speech and Visual Form, Cambridge, MA: MIT Press, pp. 362-380, 1967.

[25] Bear J., Dynamics of Fluid Flow in Porous Media, Dover, New York, 1998.

[26] P. Khayargoli, V. Loya, L.P. Lefebvre and M. Medraj, The Impact of Microstructure on the Permeability of Metal Foams, Canadian Society of Mechanical Engineering Forum, pp. 220-228, 2004.

[27] J. W. Paek, B. H. Kang, S. Y. Kim, and J. M. Hyun, Effective Thermal Conductivity and Permeability of Aluminum Foam materials, Int. J. Thermophysics, vol. 21, pp. 453-464, 2000. 
[28] K. Vafai, and C.L. Tien, Boundary and Inertial Effects on Convective Mass Transfer in Porous Media, Int. J. Heat Mass Transfer, vol. 25, pp. 1183-1190, 1982. 


\section{TABLES}

Table 1. Average effective thermal conductivity and porosity predictions.

\begin{tabular}{|cccc|}
\hline Sample & $\varepsilon_{\mathrm{v}}(\boldsymbol{\%})$ & $\mathbf{k}_{\text {eff,air }}(\mathbf{W} / \mathbf{m} . \mathbf{k})$ & $\mathbf{k}_{\text {eff,water }}(\mathbf{W} / \mathbf{m} . \mathbf{k})$ \\
$10 \mathrm{ppi}$ & 91.31 & 5.34 & 7.05 \\
$20 \mathrm{ppi}$ & 91.26 & 6.59 & 7.36 \\
$40 \mathrm{ppi}$ & 91.98 & 5.35 & 6.08 \\
\hline
\end{tabular}


Table 2. Tortuosity measurements in $x, y$ and $z$.

\begin{tabular}{|cccc|}
\hline Sample & $\boldsymbol{\tau}_{\mathbf{x}}$ & $\boldsymbol{\tau}_{\mathbf{y}}$ & $\boldsymbol{\tau}_{\mathbf{z}}$ \\
$10 \mathrm{ppi}$ & 3.31 & 3.02 & 2.26 \\
$20 \mathrm{ppi}$ & 2.66 & 2.56 & 2.58 \\
$40 \mathrm{ppi}$ & 2.54 & 2.68 & 2.15 \\
\hline
\end{tabular}


Table 3. Permeability and Ergun's coefficient predictions.

\begin{tabular}{|cccccc|}
\hline Sample & $\boldsymbol{\varepsilon}_{\mathbf{v}}(\boldsymbol{\%})$ & $\mathbf{K}_{\text {air }}\left(\mathbf{m}^{\mathbf{2}}\right)$ & $\mathbf{K}_{\text {water }}\left(\mathbf{m}^{2}\right)$ & $\mathbf{C}_{\mathbf{E}, \text { air }}$ & $\mathbf{C}_{\mathbf{E} \text {,water }}$ \\
$10 \mathrm{ppi}$ & 91.31 & $1.731 \times 10^{-7}$ & $1.705 \times 10^{-7}$ & 0.121 & 0.104 \\
$20 \mathrm{ppi}$ & 91.26 & $1.606 \times 10^{-7}$ & $1.591 \times 10^{-7}$ & 0.103 & 0.097 \\
$40 \mathrm{ppi}$ & 91.98 & $1.013 \times 10^{-7}$ & $1.029 \times 10^{-7}$ & 0.060 & 0.074 \\
\hline
\end{tabular}




\section{FIGURE CAPTIONS}

Figure 1. (a) Segmentation of filtered image, (b) island identification and removal, (c) presmoothed mesh, and (d) smoothed mesh.

Figure 2. (a) Metal foam region, and (b) pore region. An inset of the mesh for a 10 ppi foam sample is also shown.

Figure 3. Boundary conditions for the flow problem.

Figure 4. Representative volumes of (a) 10 ppi, (b) 20 ppi, and (c) 40 ppi foam sample.

Figure 5. Temperature contours in 10 ppi foam sample.

Figure 6. Effective thermal conductivity as a function of porosity with (a) air, and (b) water as the interstitial fluid.

Figure 7. (a) 10 ppi foam sample, and (b) its corresponding nodal network representation.

Figure 8. Friction factor as a function of Reynolds number for foams of different porosities: present calculations and comparisons with results from the literature.

Figure 9. Heat transfer coefficient as a function of Reynolds number with (a) air, and (b) water as the interstitial fluid.

Figure 10. (a) Flow field and temperature contours in metal foam, and (b) temperature contours in pore, shown for two cross planes perpendicular to flow direction, in 10 ppi foam sample.

Figure 11. Nusselt number as a function of Reynolds number. 


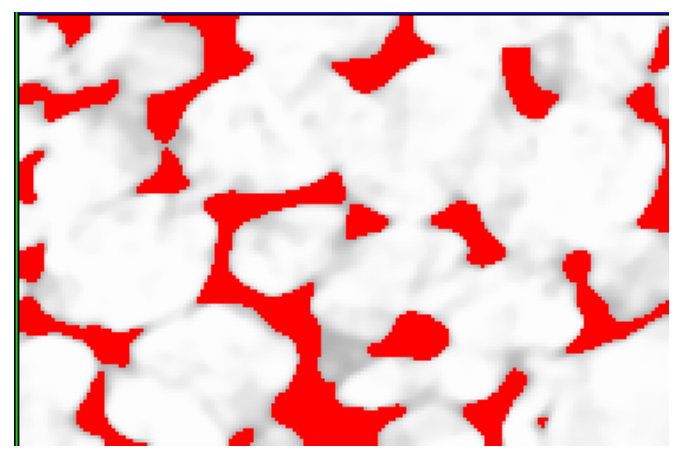

(a)

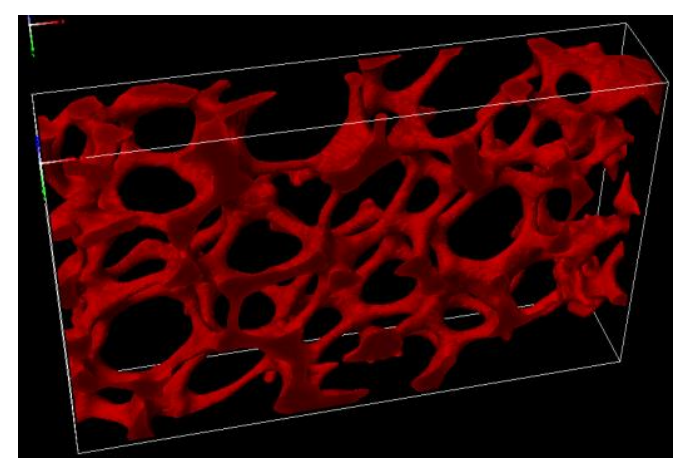

(d)

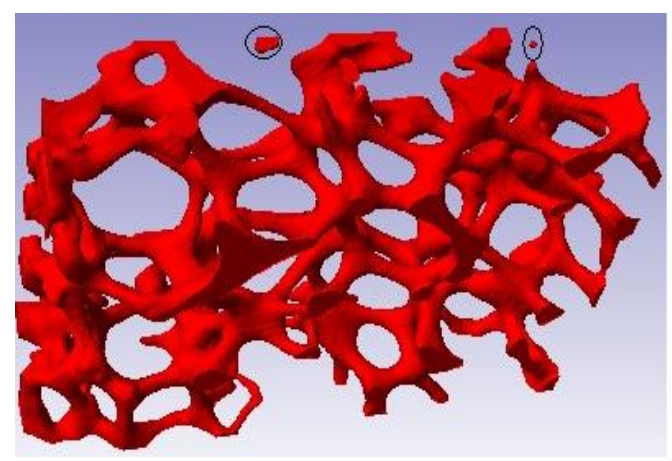

(b)

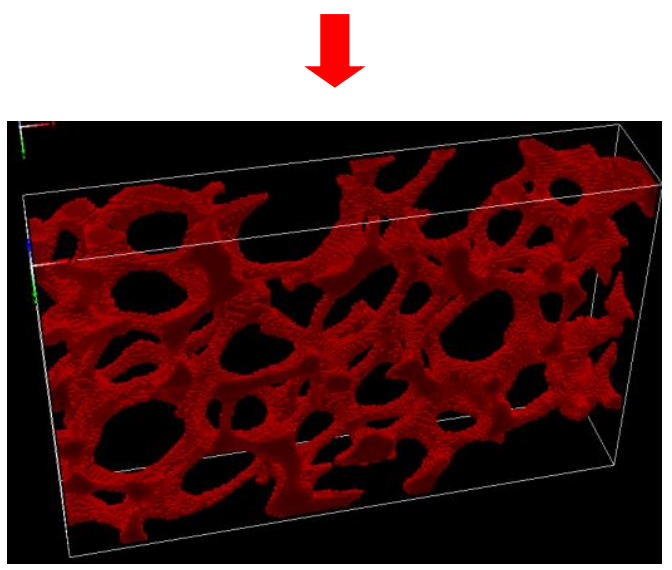

(c)

Figure 1. (a) Segmentation of filtered image, (b) island identification and removal, (c) presmoothed mesh, and (d) smoothed mesh. 


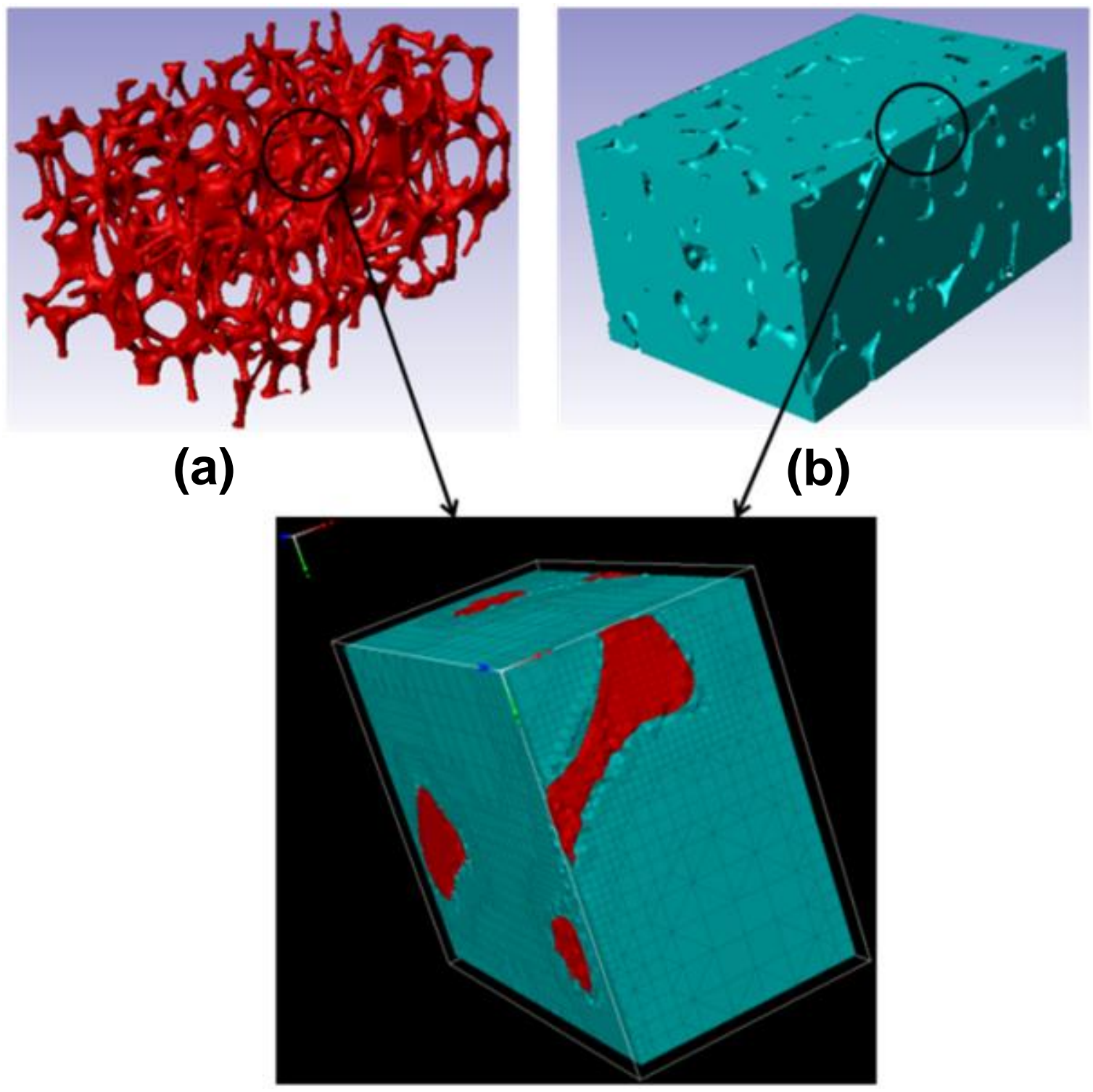

Figure 2. (a) Metal foam region, and (b) pore region. An inset of the mesh for a 10 ppi foam sample is also shown. 


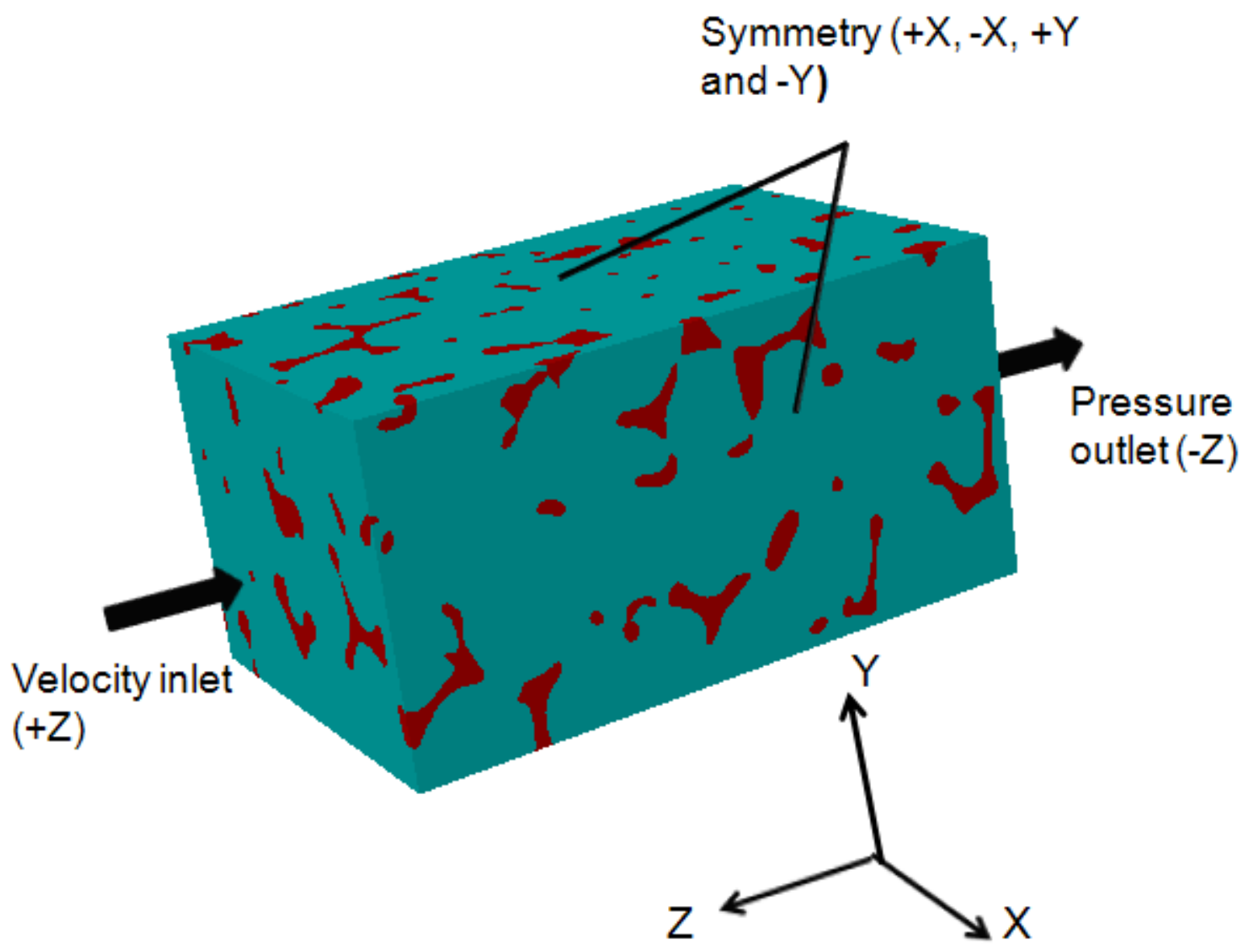

Figure 3. Boundary conditions for the flow problem. 


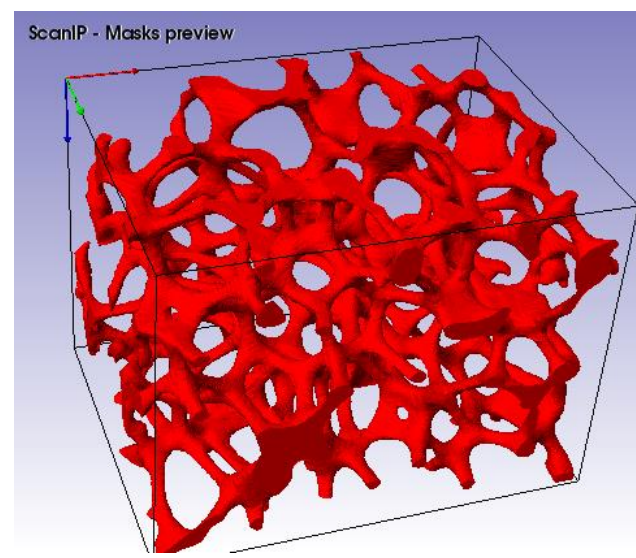

(a)

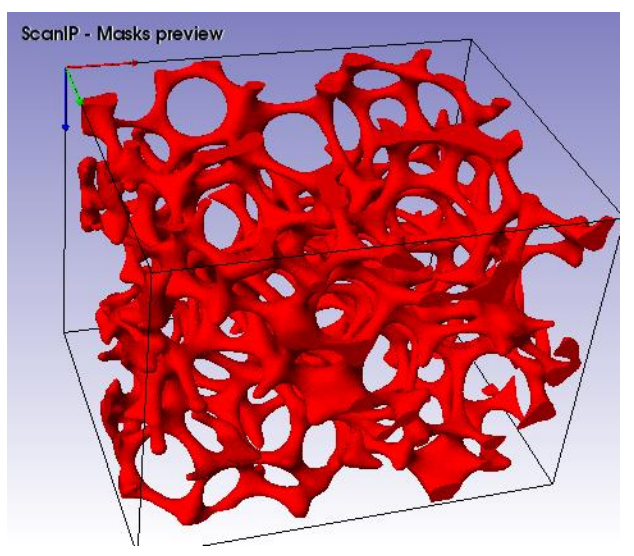

(b)

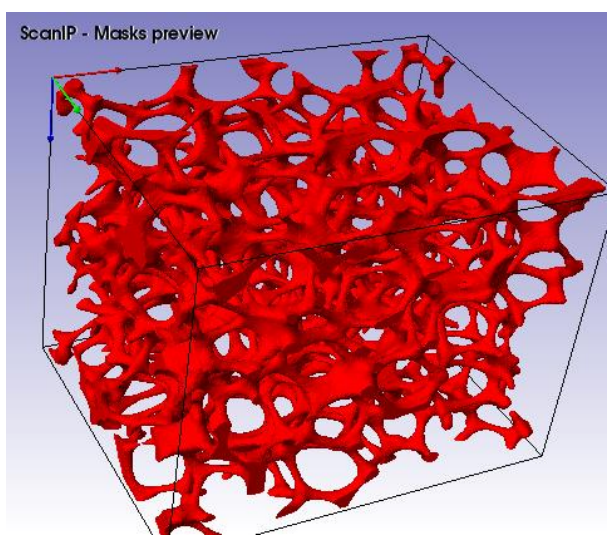

(c)

Figure 4. Representative volumes of (a) 10 ppi, (b) 20 ppi, and (c) 40 ppi foam sample. 


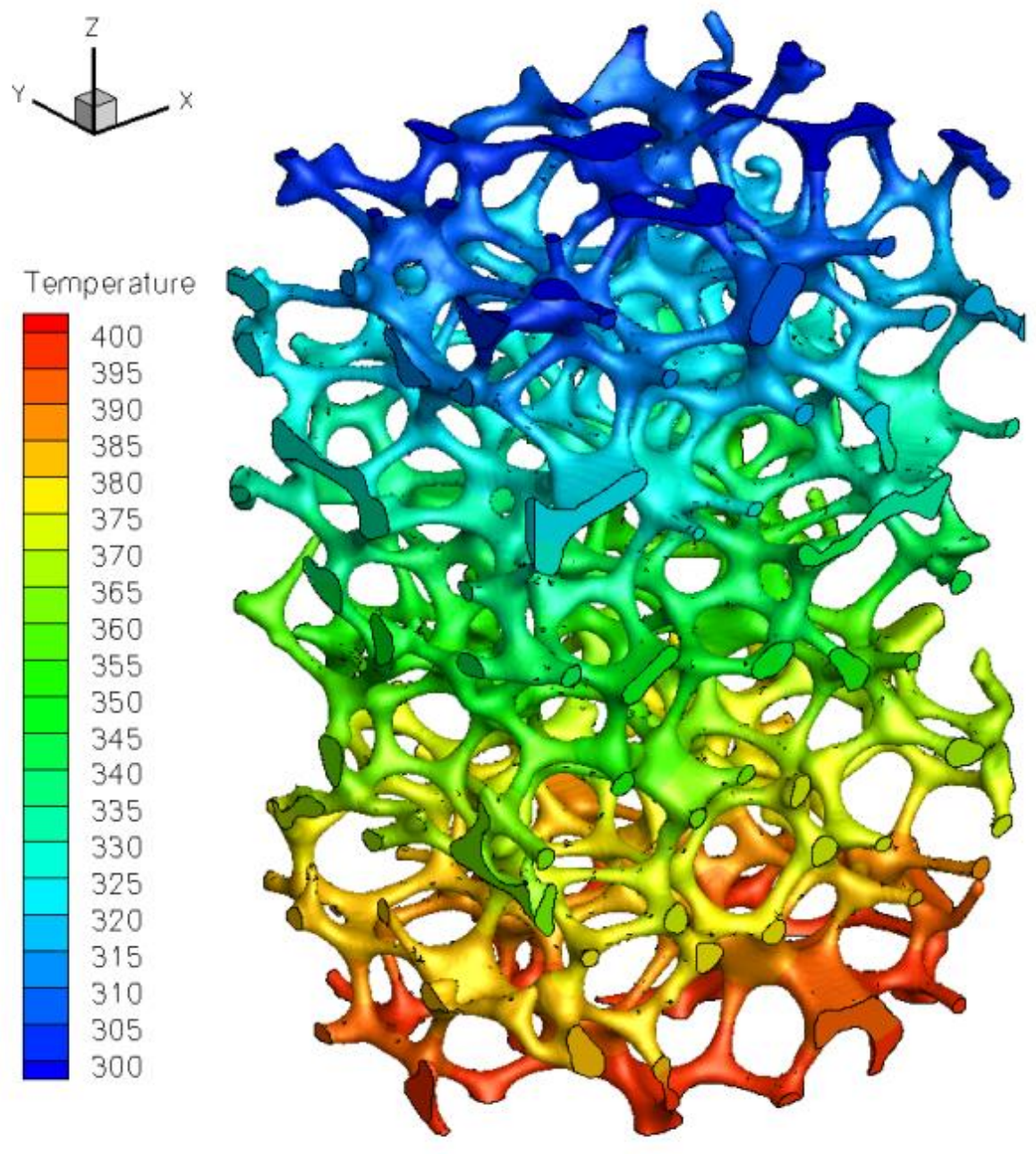

Figure 5. Temperature contours in 10 ppi foam sample. 


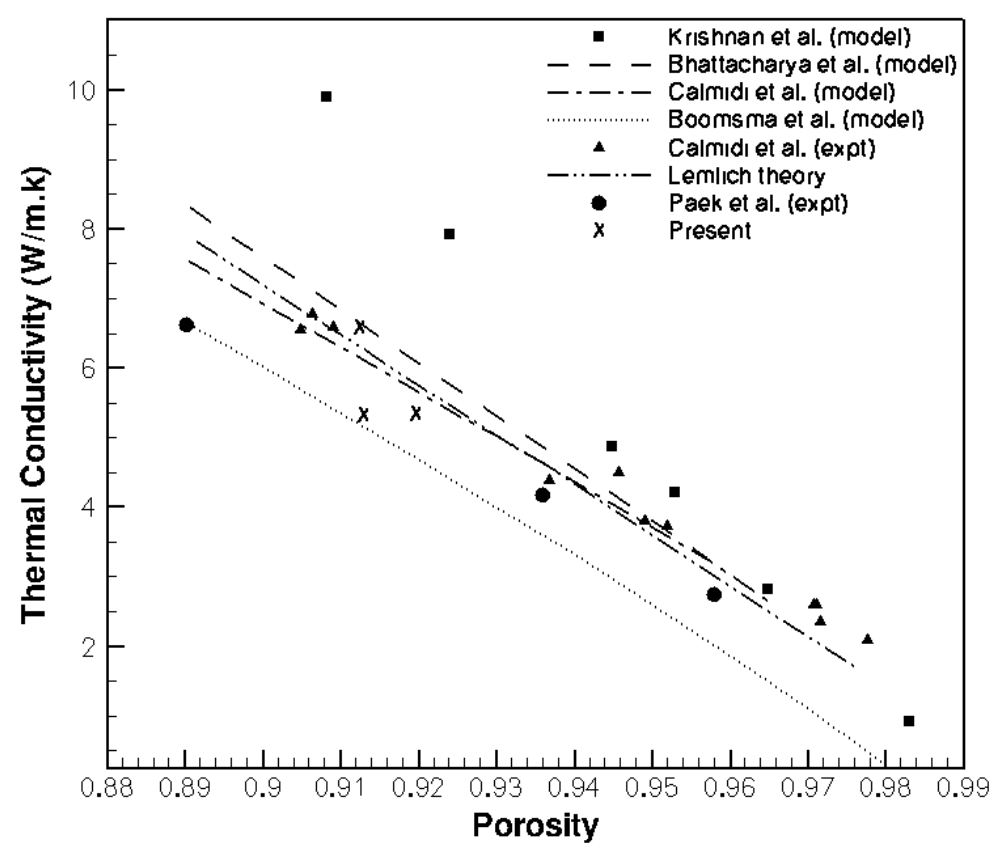

(a)

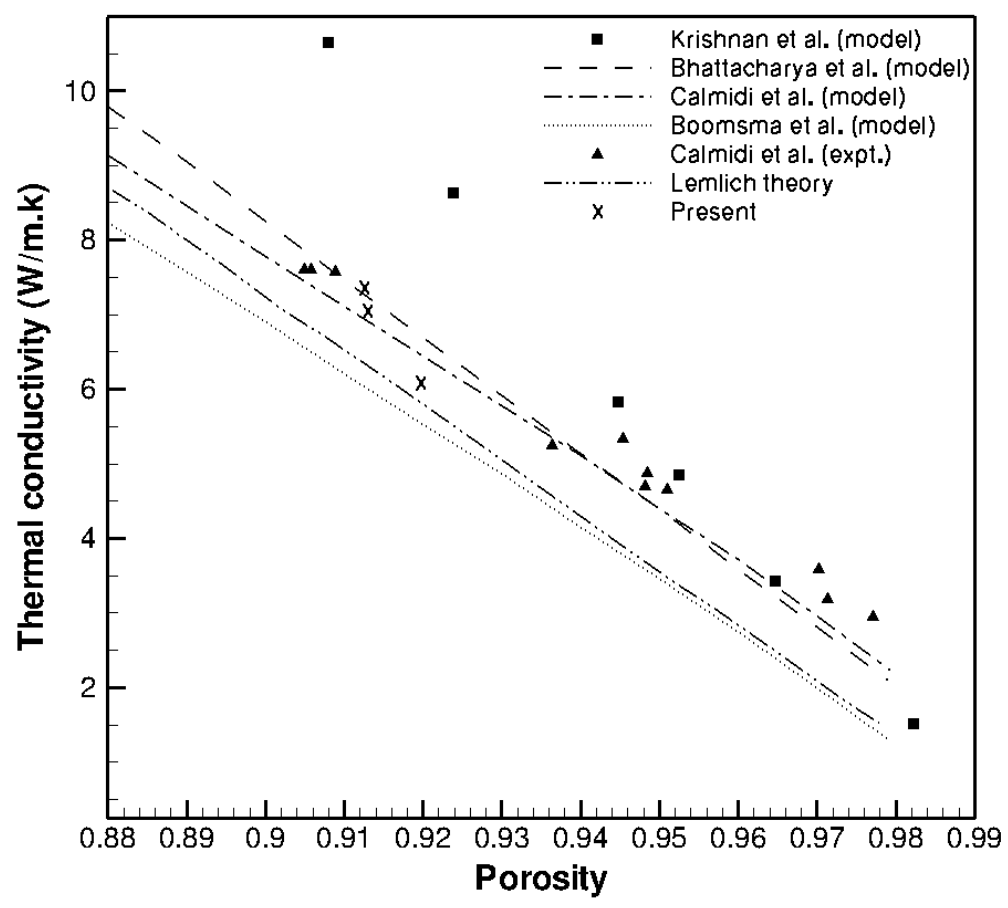

(b)

Figure 6. Effective thermal conductivity as a function of porosity with (a) air, and (b) water as the interstitial fluid. 


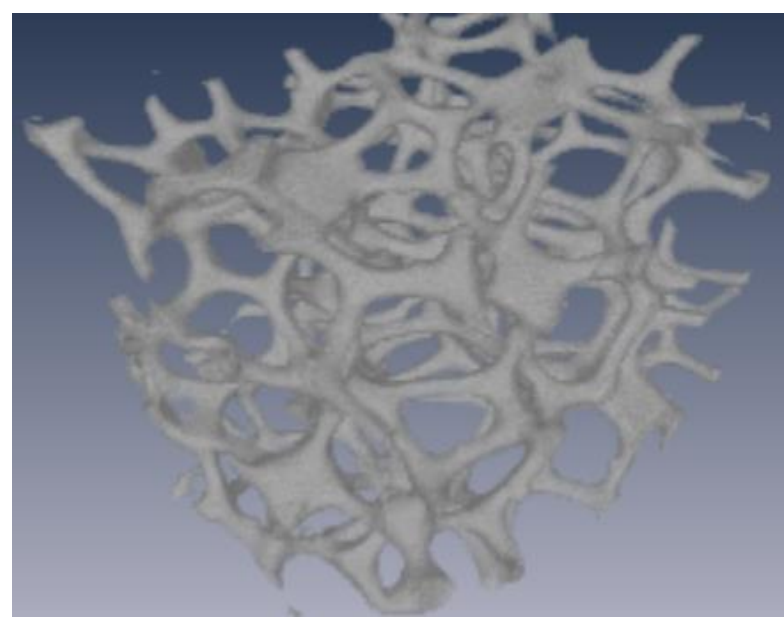

(a)

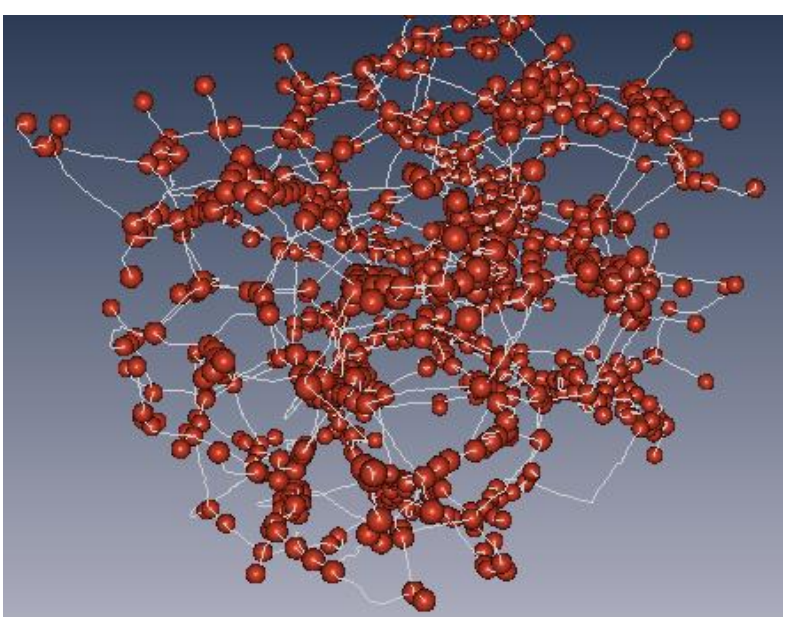

(b)

Figure 7. (a) 10 ppi foam sample, and (b) its corresponding nodal network representation. 


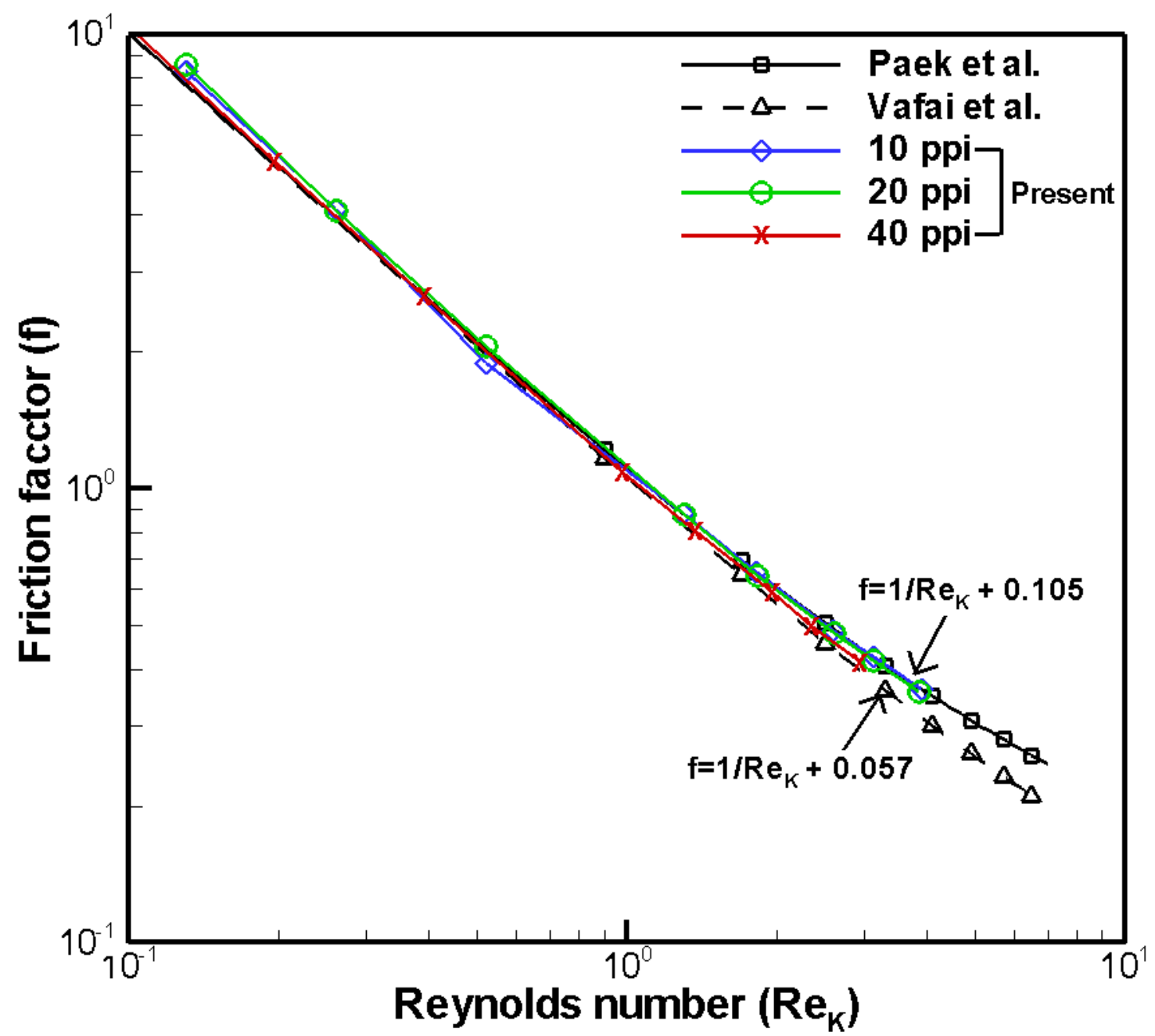

Figure 8. Friction factor as a function of Reynolds number for foams of different porosities: present calculations and comparisons with results from the literature. 


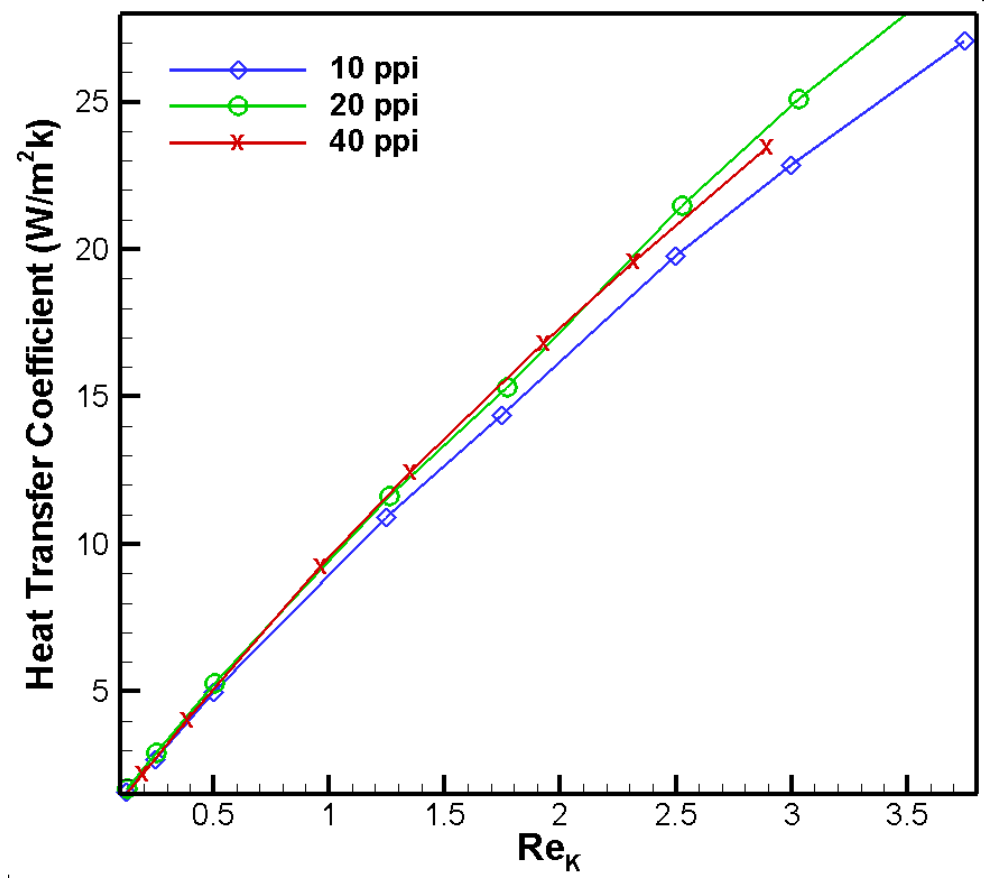

(a)

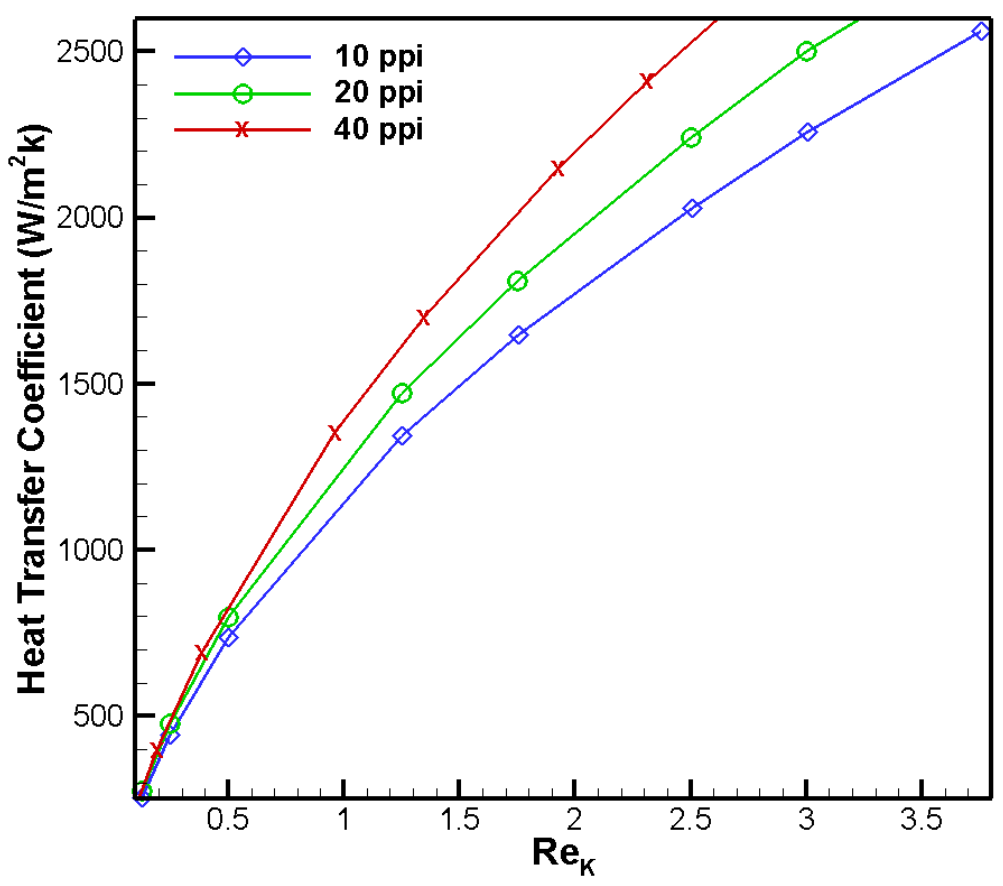

(b)

Figure 9. Heat transfer coefficient as a function of Reynolds number with (a) air, and (b) water as the interstitial fluid. 


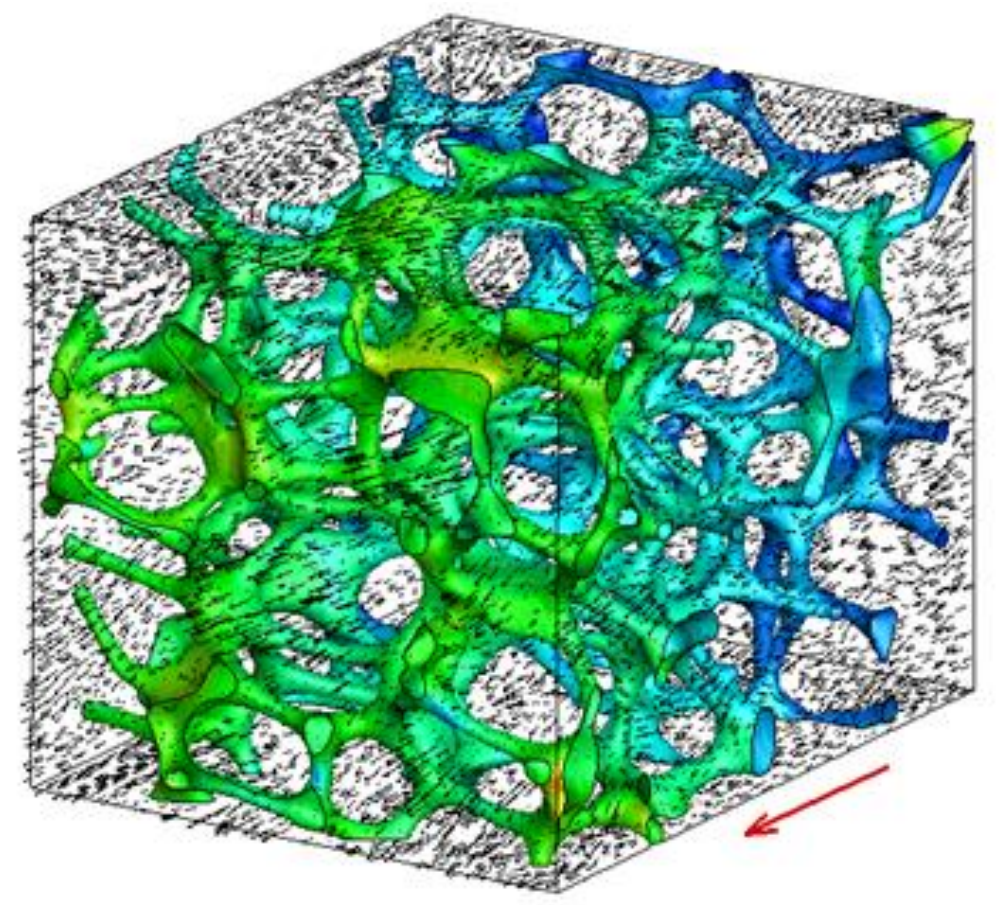

(a)
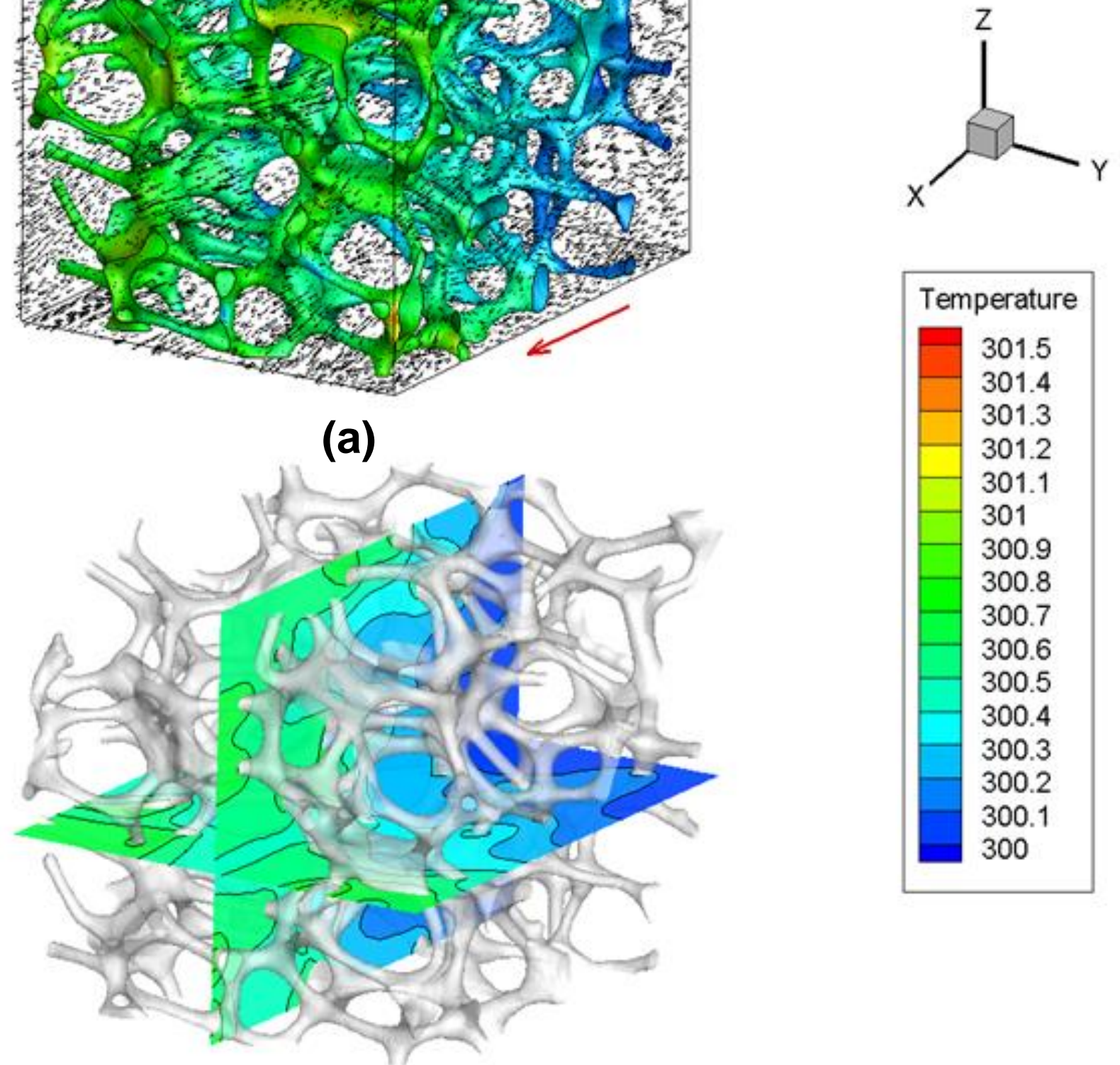

(b)

Figure 10. (a) Flow field and temperature contours in metal foam, and (b) temperature contours in pore, shown for two cross planes perpendicular to flow direction, in 10 ppi foam sample. 


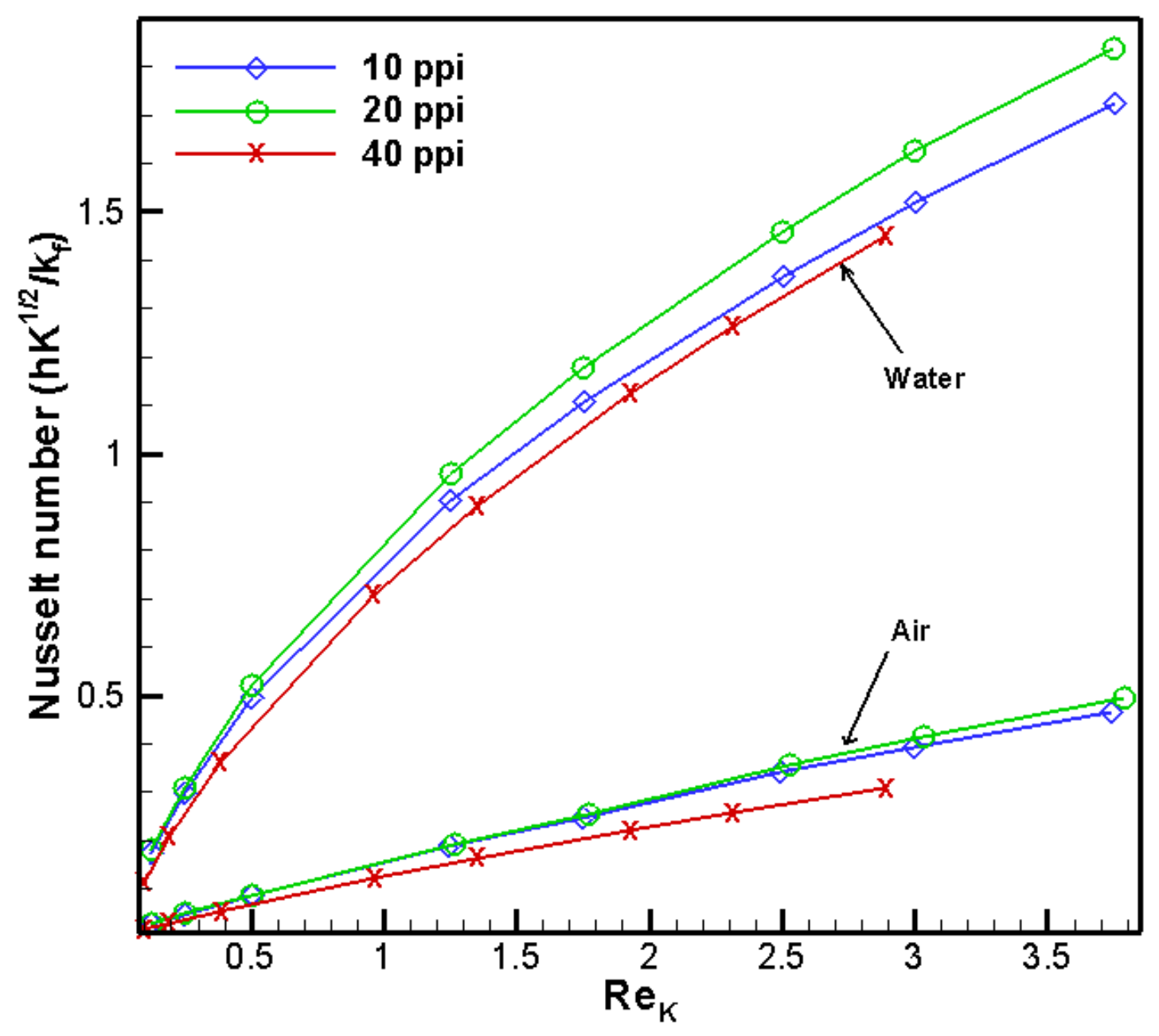

Figure 11. Nusselt number as a function of Reynolds number. 